\title{
Explorando a conexão entre a mecânica dos fluidos e a teoria cinética
}

\author{
Exploring the connection between fluid mechanics and the kinetic theory \\ Edson José Vasques, Paulo Menegasso, Mariano de Souza* \\ Departamento de Física, Instituto de Geociências e Ciências Exatas, \\ Universidade Estadual Paulista "Júlio de Mesquita Filho", Rio Claro, SP, Brasil
}

Recebido em 16 de agosto de 2015. Aceito em 30 de setembro de 2015

\begin{abstract}
O entendimento das propriedades físicas dos fluidos permitiu diversos avanços tecnológicos. Como exemplo, merece destaque a fabricação de aviões, navios e carros com perfis otimizados, os quais diminuem dramaticamente a chamada força de arrasto. São discutidos aqui os conceitos fundamentais da mecânica dos fluidos, como a força de arrasto experimentada por uma partícula se movendo num fluido viscoso proposta por Stokes em 1851, velocidade terminal, número de Reynolds e sua conexão com a teoria cinética dos gases. Fenômenos contra intuitivos, como a não variação da velocidade terminal de uma partícula se movendo sob a ação da gravidade em meios gasosos com densidades distintas para número de Reynolds baixos, são discutidos em detalhe.
\end{abstract}

Palavras-chave: escoamento laminar, velocidade terminal, lei de Stokes, número de Reynolds, teoria cinética.

The understanding of the physical properties of fluids enabled several technological advances. For instance, it is worth mentioning the production of aircrafts, ships and cars with optimized design, which reduce the co-called drag force dramatically. Here we discuss fundamental concepts of fluid mechanics, as the drag force acting on a particle moving in a viscous fluid as proposed by Stokes in 1851, terminal velocity, Reynolds number and their connection with the kinetic theory of gases. Counter-intuitive phenomena, as the insensitivity of the terminal velocity of a particle moving under the action of gravity in a medium with distinct densities for low Reynolds number, will be discussed in detail.

Keywords: laminar flow, terminal velocity, Stokes' Law, Reynolds number, kinetic theory.

\section{Introdução}

Uma das características da Terra em detrimento aos demais planetas do sistema solar se refere à existência de dois fluidos fundamentais, sem os quais a vida como a conhecemos não existiria: uma atmosfera gasosa e água no estado líquido. Daí a enorme relevância de se estudar a mecânica dos fluidos. meteorologia, hidrologia, hidráulica, oceanografia, aerodinâmica, gasdinâmica, magneto fluidodinâmica (física dos Plasmas) e reologia são alguns dos enfoques desta ciência, campo do conhecimento que se dedica ao estudo e análise das propriedades de líquidos e gases em equilíbrio ou não. A partir de

*Endereço de correspondência: mariano@rc.unesp.br conceitos básicos são desenvolvidos métodos apropriados para o entendimento e consequentemente, suas aplicações numa enorme gama de problemas como aqueles pertinentes aos campos de estudo acima mencionados 1]. Neste contexto, a mecânica dos fluidos impulsionou diversos avanços tecnólogicos. Como exemplos práticos vale mencionar a otimização do escoamento dos fluidos na indústria alimentícia, química e petrolífera, bem como o projeto de carros, aviões e navios. Olhando em uma perspectiva histórica, matemáticos, físicos e engenheiros ilustres, como Arquimedes (287-212 a.C.), que pela primeira vez estabeleceu os princípios da hidrostática, com ênfase para o empuxo e a flutuação, Leonardo da Vinci (1452-1519), que descreveu por meio de re- 
presentação e textos, muitos fenômenos envolvendo escoamentos, contribuindo assim para formar as bases iniciais da ciência da mecânica dos fluidos. Além de trabalhar nessas frentes, da Vinci projetou diversas máquinas hidráulicas. Isaac Newton (16421727) explorou vários aspectos da resistência aos escoamentos, a natureza das ondas e descobriu a contração nos jatos; Daniel Bernoulli (1700-1782), que fez inúmeras experiências e escreveu sobre o movimento dos fluidos e também organizou as técnicas manométricas de medidas e, adotando o princípio de conservação da energia, explicou o funcionamento de diversos dispositivos e descreveu a propulsão à jato; Leonard Euler (1707-1783), que explicou o papel da pressão nos escoamentos e também formulou as equações básicas do movimento, além de introduzir o conceito de cavitação e de descrever os princípios de operação das máquinas centrífugas; Joseph Louis Lagrange (1736-1813), cuja contribuição principal foram as funções potencial e corrente; Louis Marie Henri Navier (1785-1836) estendeu as equações de Euler para o movimento, incluindo as forças viscosas.

George Gabriel Stokes (1819-1903) derivou analiticamente várias relações importantes da mecânica dos fluidos, que abrangem desde a mecânica das ondas até a resistência viscosa nos escoamentos, particularmente aquela associada ao movimento das esferas num fluido. Osborne Reynolds (1842-1912) realizou experimentos em campos como cavitação, similaridade de escoamentos em rios, resistência nos escoamentos em tubulações e propôs dois parâmetros de similaridade para escoamentos viscosos, além de adaptar a equação de movimento de um fluido viscoso para as condições de escoamento turbulento 2, 3. Estes cientistas, dentre outros tantos foram fundamentais para o desenvolvimento deste fascinante campo do Conhecimento. Ainda, é relevante mencionar as contribuições de Ludwig Prandtl (1875-1953), que introduziu o conceito de camada limite e Geoffrey Ingram Taylor (1886-1975), cujos trabalhos sobre turbulência e estabilidade continuam sendo fundamentais na atualidade. Estes cientistas são considerados pais da moderna dinâmica dos fluidos [4]. Fenômenos contra intuitivos tais como a diminuição da força de arrasto com o aumento da velocidade [5], a redução na força de arrasto causada por pequenas ranhuras em uma esfera [5] e o modo como a velocidade terminal não é afetada pela densidade do fluido [5] são explorados na mecânica dos fluidos. Este último fenômeno será discutido em detalhes no que segue. Contudo, antes de passarmos para uma discussão de tais fenômenos, apresentaremos sucintamente alguns conceitos fundamentais da mecânica dos fluidos.

\section{Revisão de conceitos fundamentais}

\subsection{Tipos de escoamento}

A principal subdivisão indicada na Fig. 1 é entre escoamentos viscosos e não-viscosos. Todos os fluidos possuem viscosidade; fluidos não-viscosos, são aqueles em que as tensões de cisalhamento não estão presentes $(\eta=0)$. Estes constituem-se numa idealização, a qual simplifica a análise de problemas físicos e pode levar a resultados significativos na descrição do sistema de interesse. Da maior importância, no entanto, são os escoamentos dos fluidos viscosos, já que não há fluido real que não seja viscoso, sendo a fase superfluida do Hélio uma exceção. A subdivisão a seguir classifica os escoamentos em Laminar e Turbulento [6]. Em escoamentos laminares ocorre o deslizamento de camadas adjacentes do fluido, e nestas condições o escoamento é estacionário; já no regime turbulento as partículas do fluido se movimentam aleatoriamente, com variações abruptas de velocidade, tornando o escoamento irregular e caótico $[7,8]$. Existe uma parâmetro-chave que serve como critério para determinar se o escoamento é laminar ou turbulento, e esse parâmetro é o Número de Reynolds $(R e)$, um conceito que será discutido adiante.

\section{Velocidade terminal}

Consideremos uma esfera de massa $m$ e raio $R$ que se move sob a ação da força da gravidade $P$ e da força de arrasto $f_{a}$ exercida por um fluido com viscosidade

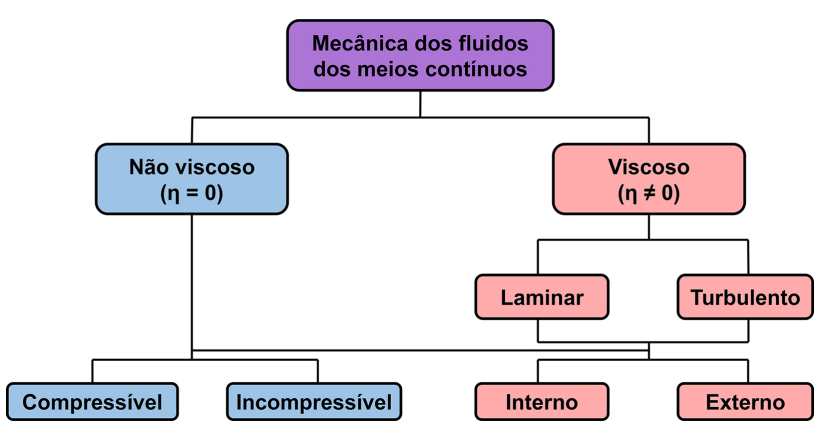

Figura 1: Subdivisões da mecânica dos fluidos proposta por Fox e McDonalds [6]. As principais divisões ocorrem na questão da viscosidade e dos escoamentos laminares e turbulentos. 
$\eta$ onde o movimento ocorre, conforme ilustrado na Fig. 2 .

Como o movimento da esfera ocorre em uma dimensão, por convenência, deixamos de utilizar aqui a notação vetorial e adotamos o eixo vertical com a orientação positiva, no sentido da força peso, que é o sentido do movimento da esfera. A equação de movimento da esfera é dada por

$$
m \frac{d v}{d t}=P-E-f_{a}
$$

onde $E$ representa a força de empuxo $E=m_{f} g$, sendo $m_{f}$ a massa de fluido deslocada pela esfera. Reescrevendo a Eq. (1), vem

$$
m \frac{d v}{d t}=\left(m-m_{f}\right) g-6 \pi R \eta v .
$$

Tal expressão é apresentada, por exemplo num dos textos de física básica [9. Antes de passarmos para a solução da equação diferencial (2), convém ressaltar que o segundo termo do lado direito da mesma foi deduzido por Stokes em 1851 [10, e é conhecida como lei de Stokes. No que segue é apresentada uma dedução desta lei, a partir das equações de Navier-Stokes.

\subsection{Lei de Stokes}

As equações de Navier-Stokes expressam o balanço, ou equilíbrio entre as forças que atuam no fluido. É importante ressaltar que este equilíbrio é mecânico,

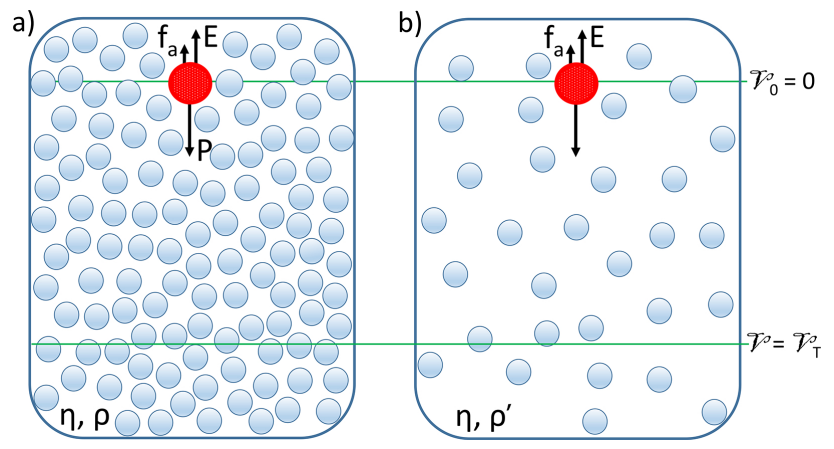

Figura 2: Esquema ilustrando o experimento realizado por Shapiro [5], no qual uma esfera (vermelha) de raio $R$ (não indicado na figura) é colocada em movimento num recipiente contendo gás (moléculas do gás são indicadas por esferas azuis) e atinge a velocidade terminal $v_{T}$, conforme discutido no texto principal. $\mathrm{O}$ meio gasoso possui viscosidade $\eta$ e diferentes densidades ( $\rho$ em a) e $\rho^{\prime}$ em b), sendo $\rho>\rho^{\prime}$ ). $P$ indica a força peso, $E$ a força de empuxo e $f_{a}$ a força de arrasto que atua na esfera (vermelha). Em a) o gás é confinado no recipiente sob pressão de 1 bar, enquanto que em b) o mesmo é confinado sob pressão de $10 \mathrm{mbar}$. ou seja, as forças de corpo (peso), as forças de superfície (pressão e forças cisalhantes) e as forças de inércia estão equilibradas. Neste artigo, as discussões sobre o equilíbrio termodinâmico dos fluidos são omitidas. Para o escoamento laminar isotérmico incompressível bi-dimensional de um fluido newtoniano (aqueles que apresentam uma relação linear entre a tensão de cisalhamento e a taxa de deformação), restrito ao caso estacionário, onde as forças de corpo são equilibradas, a equação de Navier-Stokes na sua forma vetorial é dada por

$$
\rho(\mathbf{v} . \nabla) \mathbf{v}=-\nabla p+\eta \nabla^{2} \mathbf{v},
$$

onde $\rho$ representa a massa específica do fluido (convém destacar que o termo massa específica é utilizado aqui como sinônimo do termo físico densidade), $\mathbf{v}$ sua velocidade, $p$ a pressão e $\eta$ sua viscosidade.

Conforme já mencionado anteriormente, um dos parâmetros extremamente relevante no estudo dos fenômenos envolvendo escoamento de fluidos é o Número de Reynolds Re, dado por

$$
R e=\frac{\rho v R}{\eta} .
$$

Essencialmente, tal parâmetro descreve a razão entre as forças inerciais e viscosas presentes no sistema de interesse constituindo assim um parâmetro adimensional. A partir da Eq. (4) pode-se afirmar que existem conjuntos de sistemas físicos distintos que possuem o mesmo $R$ e. Isto é possível pelo fato de que se $\rho, v, R$ e $\eta$ forem variados simultaneamente, é possível se obter o mesmo $R$ e para sistemas físicos totalmente distintos. Esta propriedade da mecânica dos fluidos possibilita por exemplo a utilização de túneis de vento para o teste de protótipos de aeronaves.

Muitos problemas físicos podem ser descritos pelo comportamento de um sistema quando um certo parâmetro ou é muito pequeno, ou muito grande [4]. Nos escoamentos para Re baixos as forças de inércia são desprezíveis, ou seja, são subdominantes, e as forças viscosas e de pressão (que atuam sobre uma partícula de fluido originada pelos gradientes de pressão dentro do fluido) são as forças dominantes e se aproximam de um balanço. Para que as equações de governo possam exibir este fato, devemos ter um pequeno parâmetro multiplicando as forças de inércia; para isto devemos adimensionalizar apropriadamente as variáveis, levando em conta a natureza dos escoamentos para $R e$ baixos, onde as forças de pressão são da ordem das forças viscosas. Para $\boldsymbol{\nabla} p$ balancear $\eta \nabla^{2} \mathbf{V}$ na Eq. (3) a variação de pressão deve ter uma ordem de grandeza de $p \sim L \eta \nabla^{2} v \sim \eta U / L$, onde $L$ é um comprimento característico e $U$ é a velocidade da corrente livre [4]. 
A adimensionalização apropriada para escoamentos com $R e$ baixos é obtida como segue

$$
\mathbf{X}^{*}=\frac{\mathbf{x}}{L}, \quad \mathbf{v}^{*}=\frac{\mathbf{v}}{U} \quad p^{*}=\frac{p-p_{\infty}}{\eta U / L} .
$$

Assim, as variações das variáveis adimensionais $\mathbf{v}^{*}$ e $p^{*}$ no campo do escoamento são da ordem da unidade, isto é, elas são normalizadas. A escala de pressão também mostra que $p$ é proporcional à viscosidade $\eta$ num escoamento com Re baixo. Substituindo a Eq. (5) na Eq. (3) obtemos a equação adimensional

$$
\operatorname{Re} \mathbf{v}^{*} \cdot \nabla \mathbf{v}^{*}=-\nabla p^{*}+\nabla^{2} \mathbf{v}^{*}
$$

No limite para $R e \rightarrow 0$, a Eq. (6) torna-se uma equação linear

$$
\nabla p=\eta \nabla^{2} \mathbf{v}
$$

onde as variáveis voltaram à sua forma dimensional, ficando evidente na Eq. (6) que o pequeno parâmetro é $R e$, onde ele multiplica o termo subdominante, as forças de inércia [4]. A Eq. (7) é a equação de governo para os escoamentos deslizantes, os quais ocorrem para baixas velocidades, altas viscosidades ou tamanho reduzido dos corpos.

A solução para o escoamento deslizante em torno de uma esfera foi primeiramente obtida por G.G. Stokes em 1851 [10]. Apresentaremos a seguir a solução elegante demonstrada por P.K. Kundu e I.M. Cohen [4, lembrando que existem outras soluções apresentadas na literatura [11 14.

Consideramos o escoamento deslizante $\left(R_{e}<<1\right)$, em torno de uma esfera de raio $a$ (ver Fig. 3) colocada numa corrente livre de velocidade uniforme $U$, conforme a Fig. 3 .

a)
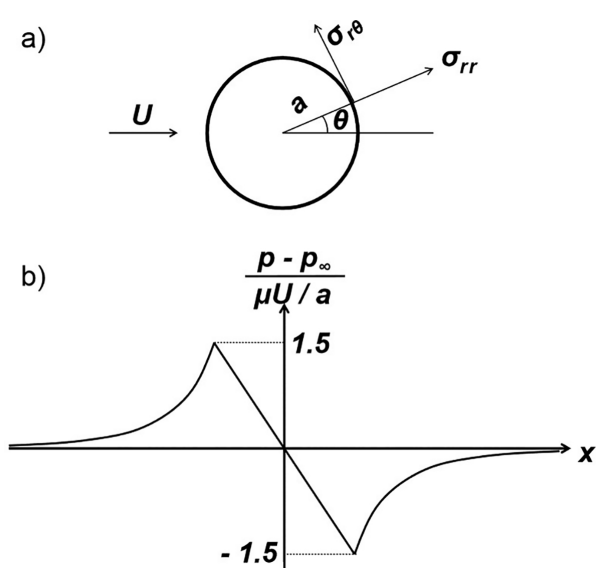

Figura 3: a) Escoamento deslizante em torno de uma esfera, onde são mostradas as componentes das tensões viscosas na superfície. b) Distribuição de pressão num plano axial ( $\varphi=$ constante), em função do deslocamento [4].
Usando o fato de que o rotacional de um gradiente é sempre zero, e que a ordem dos operadores rotacional e laplaciano pode ser intercambiada, é possível obter a equação da vorticidade ao tomar o rotacional da Eq. (7)

$$
\begin{gathered}
\boldsymbol{\nabla} \times \boldsymbol{\nabla} p=0 \\
\boldsymbol{\nabla} \times \nabla^{2} \mathbf{V}=\nabla^{2} \boldsymbol{\nabla} \times \mathbf{V}=\nabla^{2} \omega .
\end{gathered}
$$

Sendo este problema axissimétrico, a única componente da vorticidade $\vec{\omega}$ é a componente $\omega_{\varphi}$ perpendicular aos planos $\varphi=$ constante na Fig. 3. sendo

$$
\omega_{\varphi}=\frac{1}{r}\left[\frac{\partial\left(r u_{\theta}\right)}{\partial r}-\frac{\partial u_{r}}{\partial \theta}\right] .
$$

Nos escoamentos axissimétricos pode-se definir uma função corrente $\psi$ por $\mathbf{V}=-\boldsymbol{\nabla} \varphi \times \boldsymbol{\nabla} \psi$, cujas componentes da velocidade em coordenadas esféricas são

$$
u_{r}=\frac{1}{r^{2} \operatorname{sen} \theta} \frac{\partial \psi}{\partial \theta}, \quad u_{\theta}=-\frac{1}{r \operatorname{sen} \theta} \frac{\partial \psi}{\partial r} .
$$

Em termos da função corrente, a vorticidade tornase

$$
\omega_{\varphi}=-\frac{1}{r}\left[\frac{1}{\operatorname{sen} \theta} \frac{\partial^{2} \psi}{\partial r^{2}}+\frac{1}{r^{2}} \frac{\partial}{\partial \theta}\left(\frac{1}{\operatorname{sen} \theta} \frac{\partial \psi}{\partial \theta}\right)\right] .
$$

A equação de governo é

$$
\nabla^{2} \omega_{\varphi}=0
$$

Combinando as duas últimas equações, obtém-se

$$
\left[\frac{\partial^{2}}{\partial r^{2}}+\frac{\operatorname{sen} \theta}{r^{2}} \frac{\partial}{\partial \theta}\left(\frac{1}{\operatorname{sen} \theta} \frac{\partial}{\partial \theta}\right)\right]^{2} \psi=0 .
$$

As condições de contorno da Eq. (14) são

$$
\begin{gathered}
\psi(a, \theta)=0 \quad\left[u_{r}=0\right. \text { na superfície] } \\
\frac{\partial \psi}{\partial r(a, \theta)}=0 \quad\left[u_{\theta}=0 \quad\right. \text { na superfície] } \\
\psi(\infty, \theta)=\frac{1}{2} U r^{2} \operatorname{sen}^{2} \theta \\
{[\text { escoamento uniforme no } \infty]}
\end{gathered}
$$

Esta última condição é decorrente do fato de que a função corrente para um escoamento uniforme em coordenadas esféricas é $\left(\frac{1}{2}\right) U r^{2} \operatorname{sen}^{2} \theta$. 
A condição de contorno (17) sugere uma solução separável da forma

$$
\Psi=f(r) \operatorname{sen}^{2} \theta .
$$

Substituindo a Eq. (18) na Eq. (14) expandida obtém-se

$$
\begin{array}{r}
\left\{\frac{\partial^{4}}{\partial r^{4}}+\frac{\partial^{2}}{\partial r^{2}}\left[\frac{\operatorname{sen} \theta}{r^{2}} \frac{\partial}{\partial \theta}\left(\frac{1}{\operatorname{sen} \theta} \frac{\partial}{\partial \theta}\right)\right]+\right. \\
\frac{\operatorname{sen} \theta}{r^{2}} \frac{\partial}{\partial \theta}\left(\frac{1}{\operatorname{sen} \theta} \frac{\partial}{\partial \theta}\right) \frac{\partial^{2}}{\partial r^{2}}+ \\
{\left[\frac{\operatorname{sen} \theta}{r^{2}} \frac{\partial}{\partial \theta}\left(\frac{1}{\operatorname{sen} \theta} \frac{\partial}{\partial \theta}\right)\right]+} \\
\left.\left[\frac{\operatorname{sen} \theta}{r^{2}} \frac{\partial}{\partial \theta}\left(\frac{1}{\operatorname{sen} \theta} \frac{\partial}{\partial \theta}\right)\right]\right\}\left(f(r) \operatorname{sen}^{2} \theta\right)=0
\end{array}
$$

Considerando $f^{n}(r)$ a enésima derivada parcial de $f$ com relação a $r$, a equação acima implica em

$$
\begin{gathered}
\operatorname{sen}^{2} \theta f_{(r)}^{I V}-2 \operatorname{sen}^{2} \theta \frac{\partial^{2}}{\partial r^{2}}\left(\frac{f(r)}{r^{2}}\right)+ \\
+\frac{f_{(r)}^{I I}}{r^{2}}(-2) \operatorname{sen}^{2} \theta+\frac{4 f(r)}{r^{4}} \operatorname{sen}^{2} \theta=0, \\
f_{(r)}^{I V}+4 \frac{f(r)}{r^{4}}-2 \frac{f_{(r)}^{I I}}{r^{2}}- \\
2 \frac{\partial}{\partial r}\left(\frac{f_{(r)}^{I}}{r^{2}}-2 \frac{f(r)}{r^{3}}\right)=0, \\
2\left(\frac{f_{(r)}^{I I}}{r^{2}}-2 \frac{f_{(r)}^{I}}{r^{3}}-2 \frac{f_{(r)}^{I}}{r^{3}}+6 \frac{f(r)}{r^{4}}\right)=0, \\
f_{(r)}^{I V}-4 \frac{f_{(r)}^{I I}}{r^{2}}+8 \frac{f_{(r)}^{I I}}{r^{3}}-8 \frac{f(r)}{r^{4}}=0 .
\end{gathered}
$$

A solução da Eq. (23) é da forma

$$
f(r)=A r^{4}+B r^{2}+C r+\frac{D}{r} .
$$

Aplicando as condições de contorno em $\Psi(r, \theta)$

$$
\begin{aligned}
& \Psi(r, \theta)=f(r) \operatorname{sen}^{2} \theta= \\
& \left(A r^{4}+B r^{2}+C r+\frac{D}{r}\right) \operatorname{sen}^{2} \theta
\end{aligned}
$$

e usando a condição de contorno da Eq. (17), temos

$$
\begin{aligned}
& \left(A r^{4}+B r^{2}+C r+\frac{D}{r}\right) \operatorname{sen}^{2} \theta= \\
& \frac{1}{2} U r^{2} \operatorname{sen}^{2} \theta
\end{aligned}
$$

Comparando ambos os lados desta igualdade, fica evidente que devemos escolher $A=0$ e $B=\frac{1}{2} U$ e a equação para $\Psi$ fica

$$
\Psi(r, \theta)=\left(\frac{1}{2} U r^{2}+C r+\frac{D}{r}\right) \operatorname{sen}^{2} \theta .
$$

Utilizando a condição 15, ou seja, $\Psi(a, \theta)=0$,

$$
\begin{gathered}
\frac{1}{2} U a^{2}+C a+\frac{D}{a}=0, \quad \text { assim } \\
D=-\frac{1}{2} U a^{3}-C a^{2} .
\end{gathered}
$$

Utilizando agora a condição (16), ou seja, $\left.\frac{\partial \Psi}{\partial r}\right|_{r=a}=0$, tem-se

$$
U a+C-\frac{D}{a^{2}}=0,
$$

assim,

$$
D=U a^{3}+C a^{2} .
$$

Combinando as Eqa. (27) e (28), resulta em

$$
C=-\frac{3}{4} U a, \quad \text { e } \quad D=\frac{U a^{3}}{4} .
$$

Assim, a solução da Eq. (24) fica reduzida a

$$
\Psi(r, \theta)=U r^{2} \operatorname{sen}^{2} \theta\left[\frac{1}{2}-\frac{3 a}{4 r}+\frac{a^{3}}{4 r^{3}}\right] .
$$

As componentes da velocidade são obtidas das relações

$$
\begin{aligned}
& u_{r}=\frac{1}{r^{2} \operatorname{sen} \theta} \frac{\partial \Psi}{\partial \theta}=U \cos \theta\left(1-\frac{3 a}{2 r}+\frac{a^{3}}{2 r^{3}}\right), \\
& u_{\theta}=\frac{1}{r \operatorname{sen} \theta} \frac{\partial \Psi}{\partial r}=-U \operatorname{sen} \theta\left(1-\frac{3 a}{4 r}+\frac{a^{3}}{4 r^{3}}\right) .
\end{aligned}
$$

A pressão pode ser imediatamente determinada ao integrar a Eq. (7), dando como resultado

$$
p=-\frac{3 a \eta U \cos \theta}{2 r^{2}}+p_{\infty} .
$$

A distribuição de pressão é esboçada na Fig. 3. A pressão é máxima no ponto de estagnação localizado 
na esfera em frente da corrente livre, seu valor é $\frac{3 \eta U}{2 a}$, e a pressão é mínima no ponto de estagnação oposto, valendo $-\frac{3 \eta U}{2 a}$.

A determinação da força de arrasto sobre a esfera, pode ser obtida pela integração das tensões sobre a superfície da esfera. A força por unidade de área normal a superfície, sendo $\mathbf{n}$ um vetor normal unitário, é

$$
F_{i}=\tau_{i j} n_{j}=\left[-p \delta_{i j}+\sigma_{i j}\right] n_{j}=-p n_{i}+\sigma_{i j} n_{j},
$$

onde $\tau_{i j}$ é o tensor total das tensões, e $\sigma_{i j}$ é o tensor das tensões de cisalhamento (viscosas). A componente da força de arrasto por unidade de área na direção da corrente uniforme é portanto

$$
\left[-p \cos \theta+\sigma_{r r} \cos \theta-\sigma_{r \theta} \operatorname{sen} \theta\right]_{r=a}
$$

a qual pode ser entendida através da Fig. 3 .

As componentes da tensão de cisalhamento são

$$
\begin{gathered}
\sigma_{r r}=2 \eta \frac{\partial u_{r}}{\partial r}=2 \eta U \cos \theta\left[\frac{3 a}{2 r^{2}}-\frac{3 a^{3}}{2 r^{4}}\right], \\
\sigma_{r \theta}=\eta\left[r \frac{\partial}{\partial r}\left(\frac{u_{\theta}}{r}\right)+\frac{1}{r} \frac{\partial u_{r}}{\partial \theta}\right]= \\
-\frac{3 \eta U a^{3}}{2 r^{4}} \operatorname{sen} \theta
\end{gathered}
$$

de modo que a Eq. (33) torna-se

$$
\frac{3 \eta U}{2 a} \cos ^{2} \theta+0+\frac{3 \eta U}{2 a} \operatorname{sen}^{2} \theta=\frac{3 \eta U}{2 a} .
$$

A força de arrasto é obtida ao multiplicar $\frac{3 \eta U}{2 a}$ pela área da superfície da esfera, $4 \pi a^{2}$, dando portanto,

$$
f_{a}=6 \pi \eta a U
$$

conhecida como lei de Stokes para escoamento deslizante.

Fazendo a separação de variáveis na Eq. (2), definindo os limites e integrando ambos os lados, vem

$$
\int_{v_{0}}^{v} \frac{d v}{\left[v-\frac{\left(m-m_{f}\right) g}{6 \pi R \eta}\right]}=-\int_{0}^{t} \frac{6 \pi R \eta}{m} d t
$$

cujo resultado é dado por

$$
\begin{array}{r}
\ln \left[v-\frac{\left(m-m_{f}\right) g}{6 \pi R \eta}\right]- \\
\ln \left[v_{0}-\frac{\left(m-m_{f}\right) g}{6 \pi R \eta}\right]=-\frac{6 \pi R \eta}{m} t
\end{array}
$$

a qual, utilizando a definição do quociente de logarítmos, pode ser escrita como segue

$$
\ln \left\{\frac{\left[v-\frac{\left(m-m_{f}\right) g}{6 \pi R \eta}\right]}{\left[v_{0}-\frac{\left(m-m_{f}\right) g}{6 \pi R \eta}\right]}\right\}=-\frac{6 \pi R \eta}{m} t
$$

para facilitar, façamos

$$
\frac{\left(m-m_{f}\right) g}{6 \pi R \eta}=b,
$$

assim, a Eq. 40 fica

$$
\ln \left(\frac{v-b}{v_{0}-b}\right)=-\frac{6 \pi R \eta}{m} t
$$

Aplicando a definição de logarítmo, ou seja, $\left[\ln N=x \leftrightarrow e^{x}=N\right]$, temos

$$
e^{-\frac{6 \pi R \eta}{m} t}=\left(\frac{v-b}{v_{0}-b}\right)
$$

como $v_{0}=0$, chegamos a

$$
v(t)=\frac{\left(m-m_{f}\right) g}{6 \pi R \eta}\left(1-e^{-\frac{6 \pi R \eta}{m} t}\right) .
$$

Para $t \rightarrow \infty$, segue que

$$
v_{T}=\frac{\left(m-m_{f}\right) g}{6 \pi R \eta} .
$$

A Eq. 45 permite calcular a chamada "velocidade terminal" da esfera, também conhecida como velocidade limite, a qual é atingida quando a força peso e a soma do empuxo e força de arrasto são iguais, ou seja, $P=E+f_{a}$. Em tal situação, a aceleração do corpo é nula e o mesmo se move com velocidade constante $v_{T}$.

Igualando as Eqs. (44) e 45), ou seja, impondo a condição em que $v(t)$ é igual a $v_{T}$ vem

$$
\frac{\left(m-m_{f}\right) g}{6 \pi R \eta}=\frac{\left(m-m_{f}\right) g}{6 \pi R \eta}\left(1-e^{-\frac{6 \pi R \eta}{m} t}\right)
$$

Para estimar o tempo necessário para que a velocidade terminal $v_{t}$ seja atingida, expandimos a função exponencial da Eq. (43) em séries de potências. Assim, em uma aproximação de primeira ordem, obtém-se

$$
\begin{array}{r}
v(t) \simeq \frac{\left(m-m_{f}\right) g}{6 \pi R \eta}+ \\
{\left[v_{0}-\frac{\left(m-m_{f}\right) g}{6 \pi R \eta}\right] \cdot\left(1-\frac{6 \pi R \eta}{m} t\right) .}
\end{array}
$$

No estado estacionário, ou seja, $v(t)=v_{T}$, temos 


$$
0=\left[v_{0}-\frac{\left(m-m_{f}\right) g}{6 \pi R \eta}\right] \cdot\left(1-\frac{6 \pi R \eta}{m} t\right) .
$$

Contudo, apenas o segundo fator da multiplicação pode ser nulo, o que leva a

$$
1-\frac{6 \pi R \eta}{m} t=0
$$

e portanto

$$
t=\frac{m}{6 \pi R \eta} .
$$

Note que o tempo necessário para $v_{T}$ ser atingida dependa da massa e raio da esfera, bem como da viscosidade do meio. Este resultado é extremamente importante e dá origem à observação de fenômenos contra-intuitivos, como veremos na seção 3

\section{Conexão com a teoria cinética}

Estudando o movimento e as colisões de esferas perfeitamente elásticas, James Clerk Maxwell em 1860 concluiu que a viscosidade $\eta$ de um gás numa dada temperatura é independente de sua massa específica [15]; seis anos depois, ou seja, em 1866 publicou um artigo [16 onde confirmou experimentalmente o fato de a viscosidade não depender de sua massa específica.

De acordo com a teoria cinética, a viscosidade de um gás é dada por 17

$$
\eta=0,377 \cdot \frac{\sqrt{m k_{B} T}}{\sigma_{0}},
$$

onde $k_{B}$ é a constante de Boltzmann, cujo valor é $1,3806 \times 10^{-23} \mathrm{JK}^{-1}, T$ é a temperatura do gás e $\sigma_{0}$ é a secção transversal de espalhamento. A dedução da Eq. (51) é apresentada detalhadamente na Ref. 17. Tal equação nos fornece resultados notáveis: $i)$ em contraste com os líquidos, a viscosidade de um gás aumenta com a temperatura; ii) a viscosidade não depende da massa específica do líquido. Utilizando os conceitos apresentados nesta e em sessões anteriores, é discutido a seguir um fenômeno de interesse.

\subsection{Fenômenos contra intuitivos}

Um dos experimentos propostos por Shapiro [5] consiste em colocar uma esfera em movimento no interior de um tubo contendo um gás (Fig.22).

Seja uma esfera de raio $R$ e massa específica $\rho$, sua massa é definida por

$$
m=\rho . \text { Volume }=\frac{4}{3} \pi R^{3} \rho .
$$

Se $\rho_{f}$ é a massa específica do gás, então a massa do mesmo deslocada pela esfera é $m_{f}=\rho_{f}$.Volume $=$ $\frac{4}{3} \pi R^{3} \rho_{f}$, assim

$$
m-m_{f}=\frac{4}{3} \pi R^{3}\left(\rho-\rho_{f}\right) .
$$

Substituindo a Eq. (53) na Eq. (45) obtém-se

$$
v_{T}=\frac{2\left(\rho-\rho_{f}\right) R^{2} g}{9 \eta} .
$$

Substituindo a Eq. (51) na Eq. (37), temos para a força de arrasto

$$
f_{a}=6 \pi 0,377 \cdot \frac{\sqrt{m k_{B} T}}{\sigma_{0}} R v .
$$

Neste ponto, convém destacar que a Eq. (4), $R e=\rho v R / \eta$, mostra a relação entre os quatro parâmetros que caracterizam $R$ e; um destes parâmetros é $\rho$, massa específica (densidade). Re suficientemente baixos podem ser caracterizados por escoamentos com pequenas velocidades, altas viscosidades, corpos de pequenos comprimentos característicos e fluidos de baixas densidades, ou uma combinação destes parâmetros. Assim, um escoamento dito viscoso, não necessariamente precisa ser o escoamento de um fluido viscoso. Desta forma, o que vai caracterizar se o escoamento é viscoso é o fato do mesmo ter um Re suficientemente baixo.

A força de arrasto, dada pela lei de Stokes, Eq. (37), pode ser expressa em função de Re, Eq. (4), por

$$
f_{a}=\frac{6 \pi \rho R^{2} v^{2}}{R e} .
$$

Assim, enquanto a Eq. (55) nos diz que no corpo da teoria cinética dos gases a força de arrasto não depende da viscosidade do gás, e que esta, por sua vez não depende da densidade (ver Eq. (51)), a Eq. (56) nos mostra que para $R$ e baixos, conforme discutido em detalhes anteriormente, a física do sistema de interesse é governada exclusivamente pelas forças viscosas. Para deixar isto mais claro, convém destacar novamente que $R$ e pode ser definido como a razão entre as forças inerciais e forças viscosas, ou seja, $R \mathrm{e}=$ (forças inerciais) $/$ (forças viscosas). Neste contexto, a Eq. (55), em particular, nos permite entender o experimento contra-intuitivo, realizado por Shapiro [5], ilustrado na Fig. 2 e discutido a seguir. No referido experimento, Shapiro utilizou dois tubos verticais contendo ar. Em um dos tubos, o ar está à pressão atmosférica, no outro, foi extraido 99\% do ar; assim, a densidade do ar no segundo tubo é apenas $1 \%$ da densidade do ar no primeiro. A experiência consiste em deixar cair partículas de pó muito pequenas desde a parte superior de cada tubo 
e comparar suas velocidades terminais de queda. Feita tal experiência constata-se que a velocidade terminal é a mesma em ambos os tubos. Em outras palavras, o ar que foi rarefeito por um fator de 100 , oferece a mesma resistência à queda das partículas de pó no caso do ar sob pressão normal, ou ainda, o ar em ambos os tubos possui virtualmente a mesma viscosidade. Ainda, combinando as Eqs. (51) e (54) pode-se demonstrar que a velocidade terminal depende do inverso da raiz quadrada da temperatura, como segue

$$
v_{T}=\frac{2\left(\rho-\rho_{f}\right) R^{2} g \sigma_{0}}{3,393 \sqrt{m k_{B} T}} .
$$

O mesmo pode ser feito para o tempo. Assim, utilizando a Eq. (50), temos

$$
t=\frac{\sigma_{0} \sqrt{m}}{2,262 \pi R \sqrt{k_{B} T}} .
$$

Através das Eqs. (45) e (57), observa-se que a velocidade terminal da partícula permanece inalterada mediante a variação da densidade do fluido no regime de baixas densidades. Obviamente, tal afirmação deixa de ser válida no regime de fluido com densidades moderadas ou altas. Interessante notar que o tempo necessário, dado pela Eq. (58), que a esfera necessita para atingir a velocidade terminal independe da densidade do fluido. Ainda, considerando a Eq. (50) é fácil notar que o tempo necessário para que a velocidade terminal seja atingida é reduzido para fluidos mais viscosos.

\section{Conclusões}

Neste trabalho, discutimos alguns conceitos fundamentais da mecânica dos fluidos como velocidade terminal, número de Reynolds e fórmula de Stokes, para a qual é apresentada uma discussão detalhada. Uma relação entre a viscosidade e temperatura foi apresentada, estabelecendo uma conexão entre a mecânica dos fluidos e a Teoria Cinética dos Gases. Tal relação permite a demonstração de que a velocidade terminal de uma esfera se movendo sob a ação da gravidade não depende da densidade do fluido em que esta se encontra para o caso de números de Reynolds baixos. Tal situação física "contra-intuitiva", encontrada no célebre experimento proposto por Shapiro [5], discutido neste trabalho, demonstra que quando forças inerciais são desprezíveis, a densidade do fluido, que em certa medida está relacionada à sua inércia, passa a ser uma propriedade sem importância.

Convém ressaltar que a equação de Stokes é historicamente importante não apenas na mecânica dos fluidos. Ao obter a carga do elétron em seu famoso experimento da gota de óleo, Robert Millikan se utilizou da equação de Stokes. Ainda, merece destaque o fato de que Albert Einstein, na sua tese de doutorado [18], se utilizou da fórmula de Stokes no estudo do movimento Browniano. Ainda, considerando aspectos históricos a conexão entre mecânica dos fluidos e Teoria Cinética dos Gases foi proposta por Maxwell [15], quando este propôs uma relação entre viscosidade e temperatura (Eq. (51)). Tal relação permite demonstrar que a velocidade terminal de uma esfera em movimento de queda num fluido não depende da densidade do fluido, fato este que explica os resultados experimentais obtidos por Shapiro [5]. Em suma, os gases possuem uma notável propriedade, cuja predição foi um grande triunfo da Teoria Cinética: uma variação extrema na pressão não implica numa mudança significativa na viscosidade, desde que a temperatura do sistema permaneça constante.

É importante destacar que a fórmula de Stokes, dada pela Eq. (37), a qual relaciona a força de arrasto com a forma do corpo, a viscosidade e a velocidade do fluido, não só é correta com respeito à estas variáveis, mas também em relação a densidade do fluido, o que é contra intuitivo. O senso comum diria que um fluido mais denso deveria oferecer uma maior resistência ao movimento do que aquela oferecida por um fluido menos denso. No entanto, conforme demonstrado pelo experimento realizado por Shapiro 5 e discutido em detalhes neste trabalho, isto não é observado no caso de número de Reynolds baixos.

Concluindo, gostaríamos de mencionar que atualmente diversos estudos interessantes tem sido realizados no campo da mecânica dos fluidos. Como exemplo, destacamos estudos de simulações de fluxo turbulento de um fluido contendo milhares de partículas sólidas suspensas 19. Dependendo das condições de contorno estabelecidas, o mapeamento da posição e orientação de cada uma das partículas no tempo permite o entendimento do processo de erosão no solo, formação de deserto e avalanches, entre diversos outros exemplos.

\section{Agradecimentos}

Mariano de Souza agradece apoio da Fapesp (Processos No. 2011/22050-4 and 2011/17253-3) e CNPq (Processo No. 305472/2014-3), sendo a ideia de elaboração deste trabalho concebida durante visita do Prof. Vasques com uma turma de alunos do curso de Engenharia Ambiental no Laboratório de Física do Estado Sólido da Unesp - Rio Claro. Em tal ocasião, durante explicação pelo Prof. Mariano sobre o funcionamento do criostato (Projeto Fapesp No. 2011/22050-4), o Prof. Vasques mencionou a 
existência do fenômeno contra-intuitivo discutido neste artigo.

\section{Referências}

[1] R.C.C. Vieira, Atlas de Mecânica dos Fluidos (Estática, Cinemática, Fluidodinâmica) (Edgard Blücher, São Paulo, 1971).

[2] B.R. Munson, D.F. Young e T.H. Okishi, Fundamentos da Mecânica dos Fluidos (Edgard Blücher, São Paulo, 1997).

[3] H. Rouse and S. Ince, History of Hydraulics, Iowa Institute of Hydraulic Reserach, Iowa City, 1957 (Dover, New York, 1963).

[4] P.K. Kundu and I.M. Cohen, Fluid Mechanics (Elsevier Academic Press, Burlington, 2008).

[5] A.H. Shapiro, Formas y Fluidos (Eudeba, Rivadavia, 1977).

[6] R. Fox e A. McDonalds, Introdução à Mecânica dos Fluidos (LTC Editora, Rio de Janeiro, 1998).

[7] M.C. Potter, D.C. Wiggert e B.H. Ramadan, Mecânica dos Fluidos (CENGAGE Learning, São Paulo, 2015).

[8] F.W. Sears, M.W. Zemansky, H.D. Young e R.A. Freedman, Física II (Addison Wesley, São Paulo, 2003).

[9] M. Alonso, E.J. Finn, Física: Um Curso Universitário (Edgard Blücher, São Paulo, 1972), v. 1.

[10] G.G. Stokes, Transactions of the Cambridge Philosophical Society IX, p. 8 (1851).

[11] H. Lamb, Hydrodynamics (Cambridge Universty Press, New York, 1974).

[12] G.K. Batchelor, An Introduction to Fluid Dynamics (Cambridge University Press, London, 1970).

[13] M.S.D. Cattani, Elementos de Mecânica dos Fluidos (Edgard Blücher, São Paulo, 2005).

[14] F.S. Sherman, Viscous Flow (Mc Graw- Hill Publishing Company, Singapore, 1990).

[15] J.C. Maxwell, Phil. Mag. 19 (1860).

[16] J.C. Maxwell, Phil. Trans. R. Soc. 156 (1866).

[17] F. Reif, Fundamental of Statistical and Thermal Physics (Waveland Press, Inc., Long Grove, 2009).

[18] A. Einstein, Eine Neue Bestimmung der Moleküdimensionen, Bern, Buchdruckerei K. J. Wyss, (1905).

[19] A. Prosperetti, J. Fluid Mech. 768, 1 (2015). 